\title{
Tres brotes de ictiosarcotoxicosis por ingestión de Sphyraena barracuda (Walbaum) y Seriola zonata (Mitchill) en el Caribe de Colombia
}

\author{
Ricardo Alvarez
}

\begin{abstract}
Resumen
Se comentan los tres brotes de ictiosarcotoxicosis (ciguatera) conocidos en las costas colombianas sobre el Mar Caribe, causados por la ingestión de la carne de los peces Sphyraena barracuda (Walbaum) y Seriola dumerili (Mitchill).

Palabras clave: ictiosarcotoxicosis, ciguatera, Sphyraena, Seriola.

Three Ichthyosarcotoxicosis Outbreaks due to Sphyraena barracuda (Walbaum) and Seriola dumerili (Mitchill) Ingestion on the Colombian Caribbean Coast

\section{Summary}

Three reports of ichthyosarcotoxicosis (ciguatera) outbreaks on the Colombian Caribbean coast caused by fish ingestion, i.e. Sphyraena barracuda (Walbaum) and Seriola dumerili (Mitchill), are discussed.
\end{abstract}

Key words: ichthyosarcotoxicosis, ciguatera, Sphyraena, Seriola.

La ictiosarcotoxicosis o envenenamiento por ingestión de carne de pescado fue registrada por primera vez en Colombia al comprobarse que 28 personas provenientes de Taganga (Magdalena) habían desarrollado la sintomatología típica de los casos de ciguatera, una modalidad de intoxicación aséptica accidental por consumo de pescado de barracuda (Sphyraena barracuda, Walbaum) (1-3).

Esta intoxicación es frecuente en las áreas tropicales del mundo y, en particular, dentro del Mar Caribe (Cuba, Puerto Rico, República Dominicana, Venezuela, Colombia) y puede iniciarse cuando el pez se alimenta con algas planctónicas (especialmente, dinoflagelados) o bentónicas, y mariscos (moluscos, crustáceos, equinodermos, peces) que contengan la sustancia que actúa como enzima termoestable que, una vez absorbida, tiende a acumularse en el tejido muscular y en las vísceras.
La ciguatoxina es de carácter ictiosarcotóxico por lo cual se aloja en la carne, los músculos, las vísceras, los tegumentos y el moco de los pescados contaminados. En 1967, Takahachi, Tsutsumi y Yoshida comunicaron el aislamiento de la ciguatoxina y la determinación de su naturaleza química. Los investigadores lograron aislar un líquido graso, viscoso y amarillento que resistía los intentos de cristalización, agrupando sus elementos constitutivos en la fórmula $\mathrm{C}_{35} \mathrm{H}_{65} \mathrm{NO}_{8}$, que contiene un nitrógeno cuaternario y uno o varios grupos de hidroxilos (4).

Al presentarse en peces demersales de hábitos rocoso-coralinos y pelágicos de importancia comercial (pargos, meros, cabrillas, medregales, bonitos, pámpanos, carites, ojos gordos, jureles, etc.) y deportiva (dorado, barracuda, isabelitas, etc.), puede explicar los casos de intoxicación que, por lo general, no son registrados oficialmente. 
Dichas experiencias y observaciones se basan en que este tipo de intoxicación es inespecífica y se origina en peces que solo esporádicamente adquieren propiedades tóxicas; por esta razón, se consumen sin restricciones aún por personas conocedoras (ante la imposibilidad de prevenir su aparición). Por tanto, se constituye así en un problema de salud pública de sumo interés en el Caribe colombiano (1-3).

A mediados de 1984, quince personas fueron recluidas en el Hospital Universitario de Cartagena por presentar mareos, naúseas, disminución de la visión, vómitos, hiporreflexia osteotendinosa, cefaleas, gastralgia, parálisis periférica y polineuritis. Ante este cuadro clínico, se diseñó un tratamiento sintomático, con la administración de líquidos parentales, diuréticos, antineuríticos B1 y B2, y corticoides, que produjeron resultados positivos en todos los casos, no hubo fallecimientos y la mejoría progresiva se obtuvo en proporción directa a la cantidad de pescado ingerido, prolongándose la estadía de los pacientes entre un día y una semana (G. Camacho, comunicación personal).

Las personas afectadas hacian parte de una motonave, una de las cuales ejercia pesca deportiva y capturó con línea de mano, una barracuda (S. barracuda, Walbaum) de $1,20 \mathrm{~m}$ de longitud total, aproximadamente, frente a las costas del departamento de Bolívar. Cuando el barco se aproximaba a Cartagena, su puerto de destino, el tripulante fraccionó el pez y 15 de los 18 hombres de la tripulación (entre 18 y 45 años) comieron la carne del pescado en diferentes proporciones; los primeros síntomas se presentaron a las pocas horas y tan pronto se llegó al puerto se llevó a cabo la hospitalización (5).

En julio 5 de 1994, se produjo la intoxicación de dos familias al ingerir carne de Seriola zonata (medregal quimeque) de 0,4-0,5 m de longitud total capturado frente a las costas de los departamentos de Bolívar y Sucre. Las personas intoxicadas comenzaron a presentar los síntomas de la intoxicación: cefalea, diarrea, vómito, hiporreflexia osteotendinosa, urticaria, conjuntivitis, dolor y disminución de la agudeza visual. Ante el cuadro clínico, se les administraron diferentes tratamientos sintomáticos, pues, cada familia asistió a diferentes hospitales de Cartagena de Indias (Madre Bernarda y Henrique de La Vega), consistentes en líquidos parentales, diuréticos, antineuríticos B1 y B2, lavado estomacal y corticoides, así como alcohol para aliviar las molestias en las piernas (dolores y urticarias). No hubo fallecimientos y en todos los casos se obtuvieron resultados positivos con mejoría progresiva entre un día y una semana. Los más afectados fueron el hijo de la familia que originalmente ingirió el pescado, de 11 años (cefalea y disminución de la agudeza visual) y la madre de la familia vecina, de 45 años (dolor y conjuntivitis del ojo derecho), pues los síntomas se prolongaron durante una semana (6).

Tanto el pescador afectado como algunos de sus compañeros en las labores de mantenimiento y conducción de embarcaciones de pesca deportiva, afirman que estos casos de intoxicación por ingestión de pescado no son raros en el área de Cartagena y que, por lo menos, seis especies de pescados que tienen carne blanca o negra, pueden producirlas: barracudas (Sphyraena), cabrillas (Cephalopholis), chernas (Mycteroperca), dorados (Coryphaena), jureles (Caranx) y medregales (Seriola); dos especies que tienen carne negra, tunas (Thunnus) y jureles (Caranx) producen comúnmente urticarias y dos especies que tienen carne roja o rosada, sierras (Scomberomorus) y bocachicos (Prochilodus) pueden producir respuestas alérgicas. Estos testimonios comprueban y respaldan los trabajos de Martínez (1-3) y los registros del primer caso (1) y del segundo y del tercer caso $(5,6)$.

\section{Materiales y métodos}

La información que se analiza proviene de la información primaria obtenida en las entrevistas con diferentes médicos y hospitales de Cartagena de Indias (Bolívar), así como la revisión exhaustiva de la bibliografía existente en Colombia sobre ciguatera en las costas colombianas.

\section{Resultados y conclusiones}

Al comparar los brotes que se conocen hasta la fecha, Taganga (Magdalena) en una ocasión y Cartagena (Bolívar) en dos ocasiones, existen 
varias coincidencias que vale la pena resaltar: 1) la ictiosarcotoxicosis fue causada por la misma especie (S. barracuda) en dos de ellos, y 2 ) en cuanto a los sintomas, en los tres casos se presentaron naúseas, vómitos y dolores abdominales. Obviamente, las diferencias fueron marcadas y abundantes las cuales podrían explicarse en la diferente concentración de la toxina presente en algunas especies de peces, la diferente sensibilidad y edad de los afectados (niños y adultos - hombres y mujeres) en Cartagena ( $S$. zonata), en Taganga, adultos (hombres), en Cartagena ( $S$. barracuda) e incluso el tiempo de conservación y preparación del alimento (que algunas veces lo hace más severo). En realidad, aunque no se identificó la toxina de los peces, se trata de casos típicos de ciguatera cuya importancia radica en el hecho de ser transmitida por peces, algunas de cuyas especies solo esporádicamente adquieren propiedades tóxicas y, por esta razón, son consumidos sin restricciones aún por expertos, como se registró en Taganga (S. barracuda) $(1,2-5)$ y en Cartagena ( $S$. zonata) (6).

Los anteriores comentarios parecen fortalecerse con el trabajo que sobre las especies Sphyraena barracuda (barracuda), S. guachancho (picúa) y S. picudilla (picudilla) se realizó en la región de Santa Marta (Mag.), pués no se registraron efectos tóxicos en experimentos con ratones de laboratorio, ni se comentan efectos sobre las poblaciones ribereñas (7).

Las reacciones alérgicas y tóxicas en pescadores por contacto con aguas afectadas por fioraciones nocivas de fitoplancton $(8,9)$ recuerda los efectos registrados por el "manzanillo de playa" (Hyppomane mancinella) (10) sobre seres humanos. Posada-Arango (11) atribuye incluso el efecto tóxico de $S$. barracuda al consumo incidental de los frutos del H. mancinella, que caen al agua.

El síndrome característico de la ciguatera comprende manifestaciones neurológicas (parestesia, vértigo y ataxia) y gastrointestinales (diarrea, cólicos abdominales, naúseas y vómitos, también es común observar artralgia, mialgia y astenia (4). El tratamiento de la ciguatera sigue siendo problemático. Alguna ventaja podría derivarse de los lavados gástricos y de la administración de catárticos inmediata-mente después de la ingestión del pescado con el tóxico, pero ambos procedimientos suelen resultar innecesarios en razón de los vómitos provocados por la toxina. En algunos casos se ha obtenido alivio mediante la administración por vía intravenosa de gluconato de calcio al $10 \%$, pero ha habido pacientes en los cuales el medicamento resultó totalmente ineficaz. Los opiáceos se utilizan para calmar el dolor y mejorar la diarrea, y para el malestar respiratorio se recurre al oxígeno y la ventilación mecánica. La atropina parece aliviar los signos y síntomas gastrointestinales y cardiovasculares, pero carece de efectos sobre sintomas de la musculatura esquelética y solo produce una discutible mejoría en las manifestaciones neurológicas. También queda por demostrar la eficacia de las oximas (entre ellas la piridina-2-aldoxima). Los esteroides son ineficaces. Para el insomnio se administra diazepan y para el prurito de algo sirven las duchas frías y los ungüentos analgésicos (4).

No hay duda de que las ictiosarcotoxicosis accidentales no específicas, presentan un interés muy grande entre la opinión pública (12), pero también es cierto que se necesitan esfuerzos coordinados para conocer en detalle el origen, las épocas, las especies y los tratamientos. El profesor E. Balech (comunicación personal) por ejemplo, recomienda buscar los dinoflagelados bentónicos, especialmente Gambierdiscus sobre las algas del litoral colombiano, también convendría conocer si en el plancton de las aguas marinas colombianas existen Alexandrium y Pyrodinium, dos géneros productores de TPM (toxina paralizante de moluscos).

\section{Referencias}

1. Martínez, A. Ictiosarcotoxicosis: una intoxicación potencialmente frecuente, Primera parte. Rev. Tribuna Médica de Colombia 1977a; 56: 34-40.

2. Martínez, A. Ictiosarcotoxicosis: una intoxicación potencialmente frecuente. Rev. Univ. Industrial de Santander (Medicina) 1977b;7:55-94. 
3. Martínez, A. Ictiosarcotoxicosis en Colombia. Acta Médica Colombiana 1984; 9:22-27.

4. Anónimo. Los amagos de la ciguatera. Lepetit International Inc. Rev. Ressegna 1981;3:2-3.

5. Alvarez-León, R. Ictiotoxicosis ocacionada por Sphyraena barracuda (Walbaum) en el Caribe colombiano. Rev. Acta Médica de Cartagena 1993; 4 (4): 9-10.

6. Alvarez-León, R. Primer caso de ciguatera en el Caribe colombiano causado por Seriola zonata (Mitchill, 1815). Biomédica 1997;37:68-69.

7. Victoria, P. Taxonomía, osteologia, toxicidad y biología comparada del género Sphyraena (Pisces: Perciformes: Sphyraenidae) en la región de Santa Marta Magdalena, Colombia. Tesis MSc. Fac. Ciencias, Dpto. de Biología, Univ. Nal. de Colombia 1987;253 p.
8. Mercado, J.E. \& C.H. Suárez. El fitoplancton dulceacuícola, y su influencia sobre el sabor de la carne y su toxicidad en peces. INDERENA-Rev. Divulgación Pesquera 1981;17:1-10.

9. Alvarez-León, R. Efectos de las floraciones nocivas de microalgas en las zonas costeras de Colombia. Cespedesia 1998. (sometido).

10. Dao, L. Reacciones alérgicas y tóxicas al manzanillo de playa (Hippomane mancinella, L.). Rev. Consulta 1978;10-11.

11. Posada-Arango, A. Los peces, pp. 285-322 In: Estudios Científicos. Medellín (Ant.) Colombia 1909;432 p.

12. Ramírez, O. \& H. Zapata. Existe ciguatera en Cartagena? INDERENA-Rev. Manglaria 1988; 7:6-7. 